\title{
Flexibilisierung und Persuasion
}

\author{
Goranka Rocco (Trieste)
}

\begin{abstract}
The article illustrates and compares some of the persuasive strategies of the political and entrepreneurial discourse on labour market flexibilization in different contexts: within the political discourse on labour market reforms and on socio-democratic/leftist basic values, and within the entrepreneurial communication and self-presentation (job advertisements, annual relations, personnel manager guides).
\end{abstract}

\section{Flexibilisierung als Teil des wirtschaftspolitischen Paradigmenwechsels}

In der wirtschaftssoziologisch ausgerichteten Forschung und den Gouvernementalitätsstudien ist vielfach von dem seit den 1980er (z. B. USA, Großbritannien) bzw. 1990er (z. B. Deutschland) Jahren intensivierten neoliberalen Umbau die Rede (cf. Angermuller/Maeße 2014: 23, Stark 2014, Joseph 2013, Candeias 2004, Butterwegge et al. 1998 sowie Tickell/Peck 1995). In ihrer weitesten Bedeutung sind die Begriffe „neoliberale Wende“ (neoliberal turn) oder „,neoliberaler Paradigmenwechsel“1 auf unterschiedliche und in verschiedensten Feldern (z. B. Arbeit, Gesundheit, Bildung, Städtebau) stattfindende Umstrukturierungsprozesse beziehbar, für die einige gemeinsame Kernelemente angenommen werden können: fortschreitende Verschiebung im Verhältnis zwischen Staat und Markt, v. a. im Sinne einer Vermarktlichung der öffentlichen Strukturen (cf. Bosančić 2014: 35, Brand 2017: 96, Nullmeier 2004 und Chassé 2016: 370), und, damit verbunden, eine durch die „Techniken des Regierens aus der Distanz“ (cf. Angermuller/Maeße 2014: 24, Nonhoff/Herschinger 2014: 17) bzw. der „,indirekten Steuerung“ (Brand 2017: 96f.) herbeigeführte, gewinnorientierte Rationalisierung, die mit der Verlagerung der Verantwortung auf das Individuum einhergeht. Dieses soll vorzugsweise das Paradigma der permanenten kompetitivitätssteigernden Selbstoptimierung

\footnotetext{
${ }^{1}$ Dabei ist der Begriff „neoliberal“ insoweit problematisch, als die wirtschaftswissenschaftlichen, -historischen und -soziologischen Begriffsbestimmungen z. T. variieren und er darüber hinaus auch als politisches Schlagwort mit relativ diffuser Bedeutung verwendet wird. $\mathrm{Zu}$ bedenken ist ferner der übersummative Charakter des Kompositums bzw. seine potenziell irreführende Pseudotransparenz: „One of the paradoxes of neoliberalism is that it's not new and it's not liberal“, so Chomsky im Zusammenhang mit den aktuellen und vergangenen ökonomischen Machterhaltungsstrategien (Varoufakis/Chomsky 2016), ohne jedoch deshalb auf den Ausdruck zu verzichten (cf. auch Chomsky 2003). Für die Beibehaltung des Terminus spricht auch die Tatsache, dass potenzielle Ambivalenz und Resemantisierungsanfälligkeit Merkmale sind, die im Grunde viele ebenfalls als Fachbegriffe zulässige -ismen oder allgemeiner sozialwissenschaftliche Konstrukte kennzeichnen
} 
und -flexibilisierung internalisieren ${ }^{2}$ und sich im Sinne eines bestmöglichen Einsatzes der

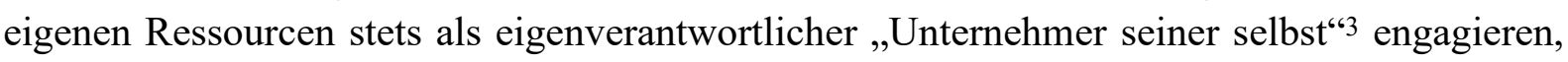
um erfolgreicher, kompetenter, effizienter, flexibler, vielseitiger, produktiver und resilienter ${ }^{4}$, insgesamt ,,(selbst)vermarktungsfähiger" zu werden und zugleich das jeweilige Sozial- und Gesundheitssystem möglichst wenig in Anspruch zu nehmen.

In der Perspektive der Soziolinguisten und Sprachanthropologen, die sich mit der Kommodifizierung auseinandersetzen, erscheinen auch die Herangehensweise an die Sprache und die jüngsten sprachpolitischen Entwicklungen als Reflexe der neoliberalen Wende (cf. z. B. Heller 2010). Zwar sind Sprachkompetenz und -verhalten zweifelsohne auch mit Blick auf andere, frühere Epochen und Systeme als Kapital auffassbar oder als „Produkte“, die, um es in bourdieuschen Termini auszudrücken, auf dem marché linguistique zu den von der Sprachgemeinschaft festgelegten, im Laufe der Sprachsozialisierung verinnerlichten „Preisen“ dargeboten werden (1984: 98). In den letzten Jahrzehnten ist jedoch verstärkt eine Tendenz zur Standardisierung, Quantifizierung und Katalogisierung dessen erkennbar, was Sprachkompetenz und Sprachenlernen sein sollen (z. B. Gemeinsamer Europäischer Referenzrahmen für Sprachen, Sprachen-Portfolio). Bezeichnend ist dabei das Paradoxon einer „uniformierten Diversität“, das im sprach-, bildungs- sowie unternehmenspolitischen (cf. Rocco/Canavese 2016: 135f.) Feiern von „Diversity“ bei gleichzeitiger Regulierung derselben besteht: Dem Hochwertkonzept stehen Diskursstrategien und institutionelle Praktiken gegenüber, die z. B. auf die Stärkung bestimmter Sprachen auf Kosten der anderen, Stärkung einer Elite-Mehrsprachigkeit, Kontrolle und Homogenisierung von Sprachkompetenzen abzielen. Kubota (2015: 25) betont in diesem Zusammenhang: „the pluralist approaches to English share with liberal and neoliberal multiculturalism the propensity to celebrate diversity but simultaneously reinforce a certain global/local commonality (e. g. English in the world), which is further propagated by neoliberal academic activities“. Flores (2013: 500) verweist auf die Parallelen zwischen dem Mehrsprachigkeitsideal und dem Ideal des neoliberalen Subjekts (hier definiert als flexibler Arbeitnehmer, lebenslanger Lerner und Performer dienstleistungsorientierter und technologischer Jobs der postfordistischen Ökonomie) und fordert eine kritischere Auseinandersetzung mit dem Mehrsprachigkeitskonzept „to avoid complicity with the promotion of a covert neoliberal agenda“" (ibd., cf. auch Piller/Cho 2013).

Im Anschluss an die skizzierten Entwicklungen, die den allumfassenden Charakter des als „,neoliberale Wende“ konzeptualisierten Paradigmenwechsels veranschaulichen, soll im Folgenden der Blick auf einen ihrer zentralen Aspekte gerichtet werden: die fortschreitende Flexibilisierung und Deregulierung des Arbeitsmarktes. Die Krise des Fordismus, die in den 1970er Jahren einsetzte und in Europa besonders seit dem Mauerfall wahrnehmbar wurde,

\footnotetext{
${ }^{2}$ Cf. dazu auch Holmes' Ausführungen zum Konzept der flexible personality (2002), die die gegenwärtige Form der Gouvernementalität, die internalisierten und kulturalisierten Muster des ,weichen“ Zwangs reflektiert, und auch Brand (2017: 96f.).

${ }^{3}$ Zu verschiedenen Aspekten der „Selbstverbetrieblichung“ bzw. Selbstökonomisierung cf. z. B. Glauser (2016), Bröckling (2007), Elster (2007), Pongratz/Voß (2003).

${ }^{4}$ Zum Konzept der Resilienz von Individuen, Strukturen und Umwelt und als Governmentality-Form cf. z. B. Slaby (2016), Joseph (2013).
} 
führte $u$. a zu einer zunehmend marktorientierten und immer feineren Ausdifferenzierung der Arbeit und zur Entstehung von Berufskategorien, die im Hinblick auf die Arbeitsbedingungen immer weiter voneinander entfernt sind (Brygo/Cyran 2016, Accornero 2001). So stehen, grob vereinfacht, an einem Ende des Kontinuums die hochspezialisierten und hochgefragten Gewinner der Flexibilisierung, bei deren Anwerbung sich Arbeit- bzw. Auftraggeber mit guten Angeboten gegenseitig zu überbieten suchen, am anderen Ende diejenigen, die in ihrer prekaritätsbedingten Hochflexibilität und -produktivität neue Arten der Wirtschaft wie z. B. die Gig-Economy und verschiedene Ausprägungen einer „Ökonomie der Verheißung“ (économie de promesse) ${ }^{5}$ erschließen bzw. gedeihen lassen. Die damit verbundenen institutionellen Transformationsprozesse beziehen ihre politisch-rechtliche Legitimation aus den teils fast unauffälligen, häppchenweise stattfindenden, teils aber als einschneidend empfundenen und politisch-medial fokussierten Arbeitsmarktreformen. Die Frage, die sich dabei aus diskurs- und persuasionsanalytischer und zugleich auch gouvernementalitätsanalytischer Perspektive stellt, ist, inwieweit der skizzierte Paradigmenwechsel mit einem allmählichen Wandel von Grundeinstellungen bzw. grundlegenden Verhaltensdispositionen einhergeht - oder auch einem Ideologiewandel, wenn wir Ideologien mit Lüdi (2007: 22) als strukturierte „Mengen von kollektiven Vorstellungen bzw. Glaubensinhalten" verstehen, die zugleich (sprach)verhaltensstrukturierend wirken und wie sich dieser sprachlich manifestiert. Dazu gehören die Fragen, welche sprachlichen Strategien in der politischen und großunternehmerischen Durchsetzung der Deregulierung und in der Konstruktion des eigenverantwortlichen, flexiblen und resilienten Mitarbeiters und/oder Bürgers eingesetzt werden, wie sich die verinnerlichten Topoi der dominanten Diskursstränge auf individueller Ebene widerspiegeln, ob es interlingual und international Parallelen gibt und ob über die Einzelsprachen hinaus Strukturen bzw. Strategien mit besonderem Persuasionspotenzial identifiziert werden können. Es handelt sich hierbei teils um Desiderata der vergleichenden Diskurslinguistik (Rocco/Schafroth 2019) und teils um Fragestellungen, deren eingehende Behandlung den Rahmen dieses Beitrags zweifelsohne sprengen würde - nicht nur aufgrund der Vielschichtigkeit der skizzierten Entwicklungen, sondern auch aufgrund der ambivalenten Rolle der Sprache (cf. Mulholland 1994: 14), die zugleich ihr Symptom und Mittel ist. Nichtsdestoweniger kann im Folgenden versucht werden, einige sprachliche Strategien und Reflexe der neoliberalen Wende im anvisierten Bereich zu illustrieren. ${ }^{6}$ Veranschaulicht werden dabei insbesondere die Sprachstrukturen und -strategien, die traditionell mit besonderem Persuasionspotenzial in Verbindung gebracht werden (z. B. Metaphern, Topoi, Resemantisierung, Euphemismen, Dysphemismen), ohne jedoch unauffälligere, und nicht unbedingt wirkungsärmere morphosyntaktische Strukturen

\footnotetext{
${ }^{5}$ Fumagalli (2016) sowie Bascetta et al. (2015) verwenden den Begriff im Sinne eines Wirtschaftens, das auf dem Arbeitsengagement derjenigen beruht, die auf eine Verbesserung der aktuellen Arbeitbedingungen hoffen (u. a. Personen mit ,atypischen” Arbeitsverträgen, Praktikanten, ehrenamtliche Mitarbeiter).

${ }^{6}$ Im Rahmen dieser Zielsetzung skizziert der vorliegende Aufsatz einige Forschungsergebnisse zum politischen und unternehmerischen Flexibilisierungsdiskurs und versteht sich zugleich als Beitrag zu den diskursanalytischen Fragestellungen des von der Verfasserin geleiteten Projekts zum Arbeitsmarkt für Akademiker im Bereich Sprachmittlung ("Mercato del lavoro per mediatori linguistici neolaureati: inserimento, prospettive, problematiche", Projektfinanzierung Università degli Studi di Trieste - Finanziamento di Ateneo per progetti di ricerca scientifica - FRA 2016, 1.1.2017-31.12.2018).
} 
aus dem Blick zu verlieren. Unabhängig davon, ob im Sinne der klassischen Rhetorik docere, movere und delectare als gemeinsam wirkende Bestandteile der persuasio betrachtet werden (cf. Lausberg 1973: § 293, Mortara Garavelli 2008: 66) oder aber Persuasion funktional als Oberbegriff (cf. Bartoszewicz 2008: 63) oder als Rahmenbestimmung (cf. Hossfeld 2011: 69) für verschiedene sprachliche Handlungen des Überzeugens und Überredens verstanden wird, steht fest, dass die Sprachmittel, die persuasive Wirkung erzielen können, äußerst vielfältig sind - und daher im Folgenden nur exemplarisch dargestellt werden können.

\section{$2 \quad$ Persuasive Strategien im Flexibilisierungsdiskurs}

Als umbrella term für verschiedene Aspekte der sprachlichen Konstruktion und Durchsetzung einer deregulierenden Beschäftigungspolitik, aber auch der kritischen Auseinandersetzung mit derselben, bietet sich der Begriff „Flexibilisierungsdiskurs“ an. Wie die bisherigen Definitionsversuche zeigen, vereint dieser viele Facetten des Diskurses über Arbeitsmarkt und Arbeitsrecht, einschließlich von Konstrukten wie „Wirtschaftskrise“, „Krise des Sozialstaats“", „Arbeits(un)willigkeit", „Leistungsmissbrauch“. In Anlehnung an Promberger (2012: 37; cf. auch Nollert/Pelizzari 2008: 139) kann der Flexibilisierungsdiskurs stark vereinfachend auf zwei grundlegende Stränge oder Dimensionen zurückgeführt werden: Der eine, hier fokussierte Strang konstruiert die Flexibilisierung des Arbeitsmarkts und des Arbeitsrechts als marktwirtschaftlichen Imperativ, welchem sich alle Beteiligten am Wirtschaftsgeschehen, Individuen, Institutionen und Staat, unterordnen müssen. ${ }^{8}$ Andererseits wird die Flexibilisierung unter dem Gesichtspunkt der Auswirkungen der Prekarisierung auf Lebenskonditionen und Lebensplanung, soziale Teilhabe, Selbst- und Fremdwahrnehmung betrachtet. Im Hinblick auf die Diskurspositionen der Akteure ist bei der erstgenannten Dimension allerdings nicht nur der öffentliche politische und unternehmerische Flexibilisierungsdiskurs von Interesse, sondern auch die Frage, wie dieser von den voice (cf. Blommaert 2005) -schwachen oder -losen Komponenten der Gesellschaft verarbeitet wird, sei es im Sinne von Kritik und Widerstand (Mattoni 2016) oder von Verinnerlichung wirtschaftspolitisch dominanter Flexibilisierungsargumente. Das von Fairclough (2011: 337f.) skizzierte Beispiel eines Schwarzarbeiters, der den internalisierten Flexibilisierungsdiskurs zur Legitimierung seiner eigenen Lage heranzieht (etwa nach dem Prinzip, Unternehmen benötigten die Flexibilität der Schwarzarbeiter), zeigt einen der Aspekte dieser Fragestellung, auf die im Folgenden nicht eingegangen werden kann, die jedoch ein dringendes Desiderat bildet.

Zum Forschungsstand bleibt noch Folgendes anzumerken: Sprachwissenschaftliches Interesse an den arbeitsmarktbezogenen Aspekten des wirtschaftspolitischen Diskurses ist im Rahmen der Politolinguistik und Diskursforschung zwar durchaus vorhanden; bezeichnend für den (z. T. unauffällig verlaufenden oder vergleichsweise wenig brisanten) Paradigmenwechsel in diesem Feld ist jedoch, dass der politische und/oder unternehmerische Flexibilisierungsdiskurs im Vergleich zu anderen, besonders oft untersuchten Diskursen (z. B.

\footnotetext{
7 Zur diskursiven Krisenkonstruktion cf. z. B. Wengeler (2013), Drommler/Kuck (2013).

${ }^{8}$ Zur zunehmenden Konvergenz der großen Parteien in dieser Hinsicht und zum Flexibilisierungsdiskurs als „Teil einer weithin beobachteten Verengung des politischen Spektrums“ cf. besonders Fairclough (2011: 344).
} 
dem Migrationsdiskurs) sprachwissenschaftlich bedeutend weniger erforscht ist und bisher insgesamt eher Sozial- und Politikwissenschaft als Linguistik zu beschäftigen scheint.

\subsection{Der politische Reformdiskurs}

Veranschaulichen lassen sich einige Tendenzen des Flexibilisierungsdiskurses zunächst an den wirtschaftspolitischen Leittexten (Teubert 1998 a, b) bzw. Texten, „die die Struktur und den Verlauf des Diskurses maßgeblich beeinflußt haben“ (Busse/Teubert 1994: 14). Dazu gehören u. a. vielbeachtete politische Grundsatz- und Reformreden bzw. -texte und ihre medialen Reflexe. Bei den hier fokussierten englischen, deutschen und italienischen Reden, die im Rahmen der jeweiligen Arbeitsmarktreformen (New Deal der New-Labour-Regierung Ende der 1990er Jahre, die Hartz-Gesetze im Rahmen der deutschen Agenda 2010 Anfang der 2000er, der italienische Jobs Act um 2014/2015) gehalten wurden, handelt es sich zwar um zeitlich auseinanderliegende, in unterschiedlichen Sprachen sowie kultur- und wirtschaftspolitischen Konstellationen konzipierte Texte. Im Hinblick auf ihren pragmatischkommunikativen Rahmen oder ihre Akteure und transtextuellen Merkmale weisen sie jedoch mehrere Analogien auf: Von besonderer Bedeutung sind die Zentralität des Themas Arbeitsmarktreform bzw. -flexibilisierung und die politische Funktion des jeweiligen Redners (Tony Blair, Gerhard Schröder, Matteo Renzi) als Vorsitzender bzw. prominent(est)er Exponent der Regierung und gleichzeitig der jeweiligen Mitte-Links-Großpartei, da den Reden bzw. Äußerungen auf diese Weise Leittextcharakter zukommt. Das Konfliktpotenzial und der Bedarf an persuasiven Strategien ergeben sich z. T. aus dem latenten Glaubwürdigkeitsproblem: Die (je nach Sichtweise) als „neosozial“, „liberal“ oder „neoliberal“ bezeichnete Umgestaltung des Arbeitsmarktes wird ausgerechnet von den Parteien vorangetrieben, deren politische Grundausrichtung traditionsgemäß auf anderen, stellenweise diametral entgegengesetzten Prinzipien fußt.

In diesen Konstellationen kommt der Resemantisierung eine besonders wichtige Rolle zu. So suggeriert das in zwei Sprachen vorliegende Grundsatzpapier von Tony Blair und Gerhard Schröder mit dem Titel „Europe: The Third Way/Die Neue Mitte“ (Blair/Schröder 1999), dass mit „Gleichheit“ eigentlich „Gerechtigkeit“ gemeint war bzw. gemeint sein sollte:

(1) The promotion of social justice was sometimes confused with the imposition of equality of outcome. The result was a neglect of the importance of rewarding effort and responsibility, and the association of social democracy with conformity and mediocrity rather than the celebration of creativity, diversity and excellence. Work was burdened with ever higher costs.

(Blair/Schröder 1999, I, englische Textfassung; Hervorheb. G. R.)

In der Vergangenheit wurde die Förderung der sozialen Gerechtigkeit manchmal mit der Forderung nach Gleichheit im Ergebnis verwechselt. Letztlich wurde damit die Bedeutung von eigener Anstrengung und Verantwortung ignoriert und nicht belohnt und die soziale Demokratie mit Konformität und Mittelmäßigkeit verbunden statt mit Kreativität, Diversität und herausragender Leistung. Einseitig wurde die Arbeit immer höher mit Kosten belastet.

(Blair/Schröder 1999, I, deutsche Textfassung; Hervorheb. G. R)

Weitere im Text auftretende Begriffspaare, auf deren Revision nach dem Prinzip , $X$ ist nicht A, sondern B' das neue Wertsystem aufbauen soll, sind z. B. market economy vs. market society („We support a market economy, not a market society.“ - „Wir unterstützen eine 
Marktwirtschaft, nicht aber eine Marktgesellschaft!“9) und ideological vs. pragmatic („Ideas of what is ,left-wing' should never become an ideological straitjacket. [...] through honest well-constructed and pragmatic policies.“- „Das Verständnis dessen, was ,links’ ist, darf nicht ideologisch einengen. [...] mit Hilfe aufrichtiger, wohl konstruierter und pragmatischer Politik."). Wie die Trias aufrichtig, wohl konstruiert, pragmatisch veranschaulicht, bilden die gegensätzlich konzipierten Begriffe ihrerseits oft Äquivalenzketten, sodass positiv konnotierte Begriffsreihen (z. B. effort, responsability, creativity, diversity, excellence im Bsp. 1) in Opposition zu negativen (hier conformity, mediocrity) oder ,überholten“ Begriffen stehen.

Die legitimierende Funktion der Umwertungsstrategien ist besonders deutlich an der Stelle, die die Frage nach der Rolle des Sozialsystems thematisiert:

(2) Decent public services are a vital concern for social democrats, but social conscience cannot be measured by the level of public expenditure. The real test for society is how effectively this expenditure is used and how much it enables people to help themselves.

(Blair/Schröder 1999, I, englische Textfassung; Hervorheb. G. R.)

Qualitätvolle soziale Dienstleistungen sind ein zentrales Anliegen der Sozialdemokraten, aber soziale Gerechtigkeit läßt sich nicht an der Höhe der öffentlichen Ausgaben messen. Der wirkliche Test für die Gesellschaft ist, wie effizient diese Ausgaben genutzt werden und inwieweit sie die Menschen in die Lage versetzen, sich selbst zu helfen.

(Blair/Schröder 1999, I, deutsche Textfassung; Hervorheb. G. R.)

Im Zusammenhang mit den Begriffspaaren bzw. -oppositionen ist auch eine Reihe reformbezogener Schlagwörter und Slogans zu erwähnen, die den englisch- und später deutschsprachigen Reformdiskurs prägten und deren zweigliedrige Struktur die latente Opposition zwischen Ansprüchen und der ,aktivierenden” Rolle des Staates thematisiert: Flexicurity, Workfare, From Welfare to Work, foster and demand, Fördern und Fordern, keine Leistung ohne Gegenleistung, Hilfe zur Selbsthilfe, neue Balance zwischen Rechten und Pflichten. Sie vermitteln zusammen mit den Syntagmen activating state, activation policy, aktivierender (Sozial)staat, aktivierende Arbeitsmarktpolitik das Konzept einer neuen Verantwortungsteilung zwischen Gesellschaft und Staat (Oschmiansky 2010), in der sich der Staat unter dem Motto „Third Way“ bzw. „Neue Mitte“ jenseits der neoliberalen Strategien, aber auch jenseits des traditionellen Wohlfahrtstaatlichkeitskonzepts als „neo-sozial“ zu positionieren sucht (Trube 2004: 62f.). Im Einklang damit wird bereits in Schröders Regierungserklärung 2002 ,eine neue Kultur der Selbstständigkeit und der geteilten Verantwortung“ heraufbeschworen (Oschmiansky 2010). Zweigliedrige, nach dem Prinzip der Antonymie funktionierende Syntagmen findet man allerdings auch im flexibilisierungskritischen Diskurs; ein Beispiel stellt der um die Jahrhundertwende oft benutzte und danach noch sporadisch auftretende ironisch-sarkastische Dysphemismus Genosse der Bosse dar.

In den späteren Reformreden von Gerhard Schröder (2003) und Matteo Renzi (2014) begegnet man antagonistischen Begriffsketten, die ähnlich funktionieren: So bilden im folgenden Ausschnitt aus der Agenda-Rede (Bsp. 3) Bürokratie, belasten, verbauen und die durch die Anadiplose amplifizierte Dublette Ansprüche und Leistungen eine Äquivalenzkette,

\footnotetext{
${ }^{9}$ Das Ausrufezeichen ist nur in der deutschen Fassung zu finden.
} 
welche als Negativkorrelat der Begriffskette die Jüngeren und Zukunftschancen gegenübersteht. Zusätzlich legitimiert die Unvermeidbarkeitsrhetorik (es wird unausweichlich nötig sein) den geplanten Sozialabbau. ${ }^{10}$ Bezeichnenderweise lassen sich im Kontext des Thatcherismus ähnliche Tendenzen nachweisen, so verweist Marchart $(2013: 149,170)$ auf die ,,antagonistische Äquivalenzkette von /Bürokratie/ = /Abhängigkeit/ = /Kollektivismus/ = /Staat/“, „deren einzelne Signifikanten in einer Kontraritätsrelation zu protagonistischen Signifikanten wie $/$ Individualismus/ = /nitiative/ $=/$ Konkurrenz/ $=/$ Freiheit/ stehen“ (S. 170).

(3) Wir werden erhebliche Einsparungen durch Umstrukturierungen im System und durch Abbau von Bürokratie erreichen. Aber es wird unausweichlich nötig sein, Ansprüche und Leistungen zu streichen, Ansprüche und Leistungen, die schon heute die Jüngeren über Gebühr belasten und unserem Land Zukunftschancen verbauen.

(Agenda-Rede, 2013)

Das Schlagwort Bürokratie tritt in der erwähnten Rede auch an mehreren anderen Textstellen in Opposition zu positiv konnotierten Begriffen und Begriffsreihen, zu denen u. a. frei, befreien, modernisieren, verschlanken gehören. In Matteo Renzis Ausführungen zum Jobs Act ${ }^{11}$ nimmt Bürokratie bzw. Bürokratisierung stellenweise geradezu monsterhafte Züge an (wie es davor vor allem in den Reden liberaler Politiker beobachtet werden konnte):

(4) Il nostro è un paese arrugginito, un paese impantanato, incatenato da una burocrazia asfissiante, da regole, norme e codicilli che paradossalmente non eliminano l'illegalità.

(Rede vor dem Senat, 2014)

Als Hauptfunktion dieser Begriffsoppositionen erweist sich eine neue, mit dem Reformprojekt kongruente Festlegung des ideologisch hochgradig polysemen Begriffs sinistra. Dieser wird, wie die nächsten beiden Auszüge veranschaulichen, durch eine Reihe von Oppositionen definiert: handeln statt ideologische Debatten führen, Wandel befürworten statt Wandel ablehnen, Arbeitnehmer verteidigen statt Totems (an anderer Stelle: Tabus) verteidigen, Ungerechtigkeit bekämpfen statt Ungerechtigkeit verteidigen.

(5) Questa riforma è di sinistra, se la sinistra serve a difendere i lavoratori e non i totem. Se serve a difendere il futuro, e non il passato. Se serve a difendere tutti, non qualcuno già garantito.

(Rede vor der Direzione Nazionale, 2014)

(6) Personalmente dico a quella parte di sinistra più dura rispetto alle necessità di cambiare le regole del gioco sul lavoro che, per come la interpreto io, la sinistra è combattere l'ingiustizia, non difenderla $[\ldots]$.

(Rede vor der Abgeordnetenkammer, 2014)

Insgesamt dient die Resemantisierung durch antagonistische Begriffspaare und Äquivalenzketten neben anderen Varianten des Kampfes um Wörter ${ }^{12}$ einem „Restyling“ der

\footnotetext{
10 Für weitere Persuasionsstrategien der Agenda-Rede und anderer Reformreden aus dem Jahr 2003 cf. Schröter (2004), Hegelich/Knollmann/Kuhlmann (2011), Rocco (2015b: 87-95).

11 Im Folgenden werden vier Reden von Matteo Renzi zitiert: vor dem Senat am 24. Februar 2014, vor der Abgeordnetenkammer am 16. September 2014, auf der Tagung der Direzione nazionale des Partito Democratico am 29. September 2014 und auf der Großveranstaltung Leopolda 5 am 25. Oktober 2014.

12 Zum Kampf um sozialdemokratische und christdemokratische Fahnenwörter cf. Hermanns (1989).
} 
öffentlichen Selbstdarstellung, das auch durch andere Persuasionsstrategien erfolgen kann. So zeugt die folgende Äußerung des damaligen SPD-Fraktionsvorsitzenden Struck von einer Art philosophisch-anthropologischem Gesinnungswandel in den eigenen Reihen: „Es war zu optimistisch anzunehmen, dass Menschen das System nur in Anspruch nehmen, wenn sie es wirklich brauchen".13 Gleichzeitig tastet sie sich an ein bis $\mathrm{zu}$ diesem Zeitpunkt im öffentlichen SPD-Diskurs eher tabuisiertes Thema (Klein 2007: 225-227) heran, das sich jedoch im Kontext der Eingriffe in das Sozialsystem als durchaus funktional erweist: die Frage des Missbrauchs von Sozialleistungen.

In der zitierten Aussage Strucks sowie in der Agenda-Rede Schröders wird auf potenzielle „Sozialschmarotzer“ allerdings nur vorsichtig verwiesen (in der Agenda-Rede z. B. durch Pseudoeuphemismen bzw. euphemistisch formulierte Wendungen mit dysphemischer Wirkung wie „Menschen, denen wir mehr abverlangen müssen“, „Menschen, die länger als zwölf Monate arbeitslos sind“", cf. Rocco 2015b: 91f.). ${ }^{14}$ Doch in anderen Zusammenhängen und Textsorten erweist sich, dass der stigmatisierende Topos ${ }^{15}$ durchaus den Weg in die öffentliche Kommunikation gefunden hat: So enthält die „Broschüre des Bundesministeriums für Wirtschaft und Arbeit" (2005) bereits im Titel, in dem vom damaligen Wirtschaftsminister Clement signierten Vorwort sowie im übrigen Text mehrere (z. T. metaphorische) Schlagwörter und Begriffsoppositionen der Hartz-IV-Diskussion: „Vorrang für die Anständigen. Gegen Missbrauch, ,Abzocke' und Selbstbedienung im Sozialstaat“. Weitere Schlagwörter und Wendungen mit hohem dysphemischen Potenzial können an folgenden Textausschnitten veranschaulicht werden: „Die Hemmschwelle für Sozialbetrug ist offensichtlich bei Einigen gesunken“, „Sozialleistungen zu erschleichen versuchen“, „Mitnahme-Mentalität“", „Jeder Euro, der am Arbeitsmarkt ,abgezockt“ wird“, „Melkkuh Sozialstaat - die alltägliche Selbstbedienung am Gemeinwohl“. Aufgegriffen werden hier auch Schlagwörter des reformfeindlichen Diskursstrangs: „Trotz mancher Kritik beharre ich darauf: Die Arbeitsmarktreform ist alles andere als ,sozialer Kahlschlag' oder ,Armut per Gesetz'“.

Dass auf dem Sozialmissbrauch-Topos beruhende Schlagwörter wie Hartz-IV-Abzocke(r), System-Abzocke( $r$ ) (genauso wie die englischen Ausdrücke welfare scroungers, benefit cheats usw.) eine lange Karriere haben, zeigen neben zahlreichen Beiträgen in Internetforen mehrere Zeitungstitel, die z. T. Jahre nach den Reformen entstanden sind, etwa „Deutschland frechster Hartz-IV-Abzocker“ (Bild, 6.12.12), „Jobcenter sollen Hartz-IV-Abzocker härter bestrafen“

\footnotetext{
13 Aussage des SPD-Fraktionsvorsitzenden Peter Struck, Aachener Nachrichten vom 26.06.2006; zit. nach Klein (2007: 227).

${ }^{14}$ Latent vorhanden ist der Vorwurf des Sozialmissbrauchs auch in den oben erwähnten Schlagwörtern wie activation policy, aktivierende Arbeitsmarktpolitik, die präsupponieren, dass jemand aktiviert werden kann oder sollte.

${ }^{15}$ Unter „Topoi“ werden hier und im Folgenden in Anlehnung an das Toulmin“sche Schema (1996) und einige viel rezipierte Definitionen die in die kollektiven Denkweisen eingebetteten, plausibel erscheinenden Prämissen einer Diskursgemeinschaft (Spira 1981: 42, Hossfeld 2011: 76) bzw. „wiederkehrende Argumentationsmuster” (Wengeler 1997: 96) verstanden. Als Teil des kollektiven Wissensbesitzes bzw. als endoxa reflektieren sie das, worauf ,,sich eine Diskursgemeinschaft in ihren Argumentationen abstützt” (Eggler 2006: 35). Die sich daraus ergebende persuasive Wirkung kann mit der auf den ersten Blick vage erscheinenden Beschreibung Spiras „Ein Topos ist, was einleuchtet" (Spira 1981: 42) auf den Punkt gebracht werden.
} 
(Die Welt, 2.9.16), aber auch Publikationen, die sich mit dem Stigma auf ironisch-kritische Weise auseinandersetzen:

(7) Wir sind die Parias der Gesellschaft, eigentlich deren Auswurf, umso mehr seit Hartz IV ein Schimpfwort geworden ist, gleichbedeutend mit Schmarotzer, Penner, Versager, Asozialer und was es sonst noch für freundliche Ausdrücke dafür gibt.

(Harvey 2012, Prolog)

Während sich der an die Merkmale der jeweiligen Sozialsysteme gebundene Leistungsmissbrauch-Topos im englischen und deutschen Flexibilisierungsdiskurs als reformfunktional erweist, betrifft das italienische Pendant, das eher allgemeiner als Systemmissbrauch-Topos bezeichnet werden könnte, bisher nicht in erster Linie arbeitslose Leistungsempfänger, sondern (sicher auch angesichts der strukturellen Unterschiede) eher andere Konstrukte, wie z. B. „migranti“, „extracomunitari“, „statali“. Mit Blick auf die im Reformpaket Jobs Act enthaltene Kündigungsschutz-Lockerung ${ }^{16}$ haben Gegenüberstellungen wie „serve a difendere tutti, non qualcuno già garantito“ in Bsp. 5 ein nicht zu vernachlässigendes Legitimierungspotenzial. Die auf diese Weise konstruierte Kluft zwischen der oft als unverdientes oder ungerechtes Privileg repräsentierten festen Anstellung und der Allgemeinheit (tutti) verleiht dem Deregulierungsvorhaben die Aura einer Ungerechtigkeiten mildernden Reform und blendet zugleich andere, wachsende sozioökonomische Klüfte aus.

Eine Zielscheibe im Flexibilisierungsdiskurs zum Jobs Act sind die Reformgegner aus den eigenen Reihen und aus anderen Parteien, die an dem erwähnten Artikel 18 festhalten, der den Kündigungsschutz garantiert und das diskursive Zentrum vieler Debatten bildet. Eine dysphemische Wirkung haben dabei grotesk anmutende Sprachbilder, die verschiedene Topoi des politischen Diskurses evozieren und die Ansichten dieser Gruppierungen als realitätsfern zeichnen (,Non siamo un club di filosofi ma un partito politico che decide“). Im folgenden Redeausschnitt werden sie als anachronistische Figuren karikiert, die etwa beim I-Phone fragen, wo sie die Münze einwerfen sollen, den USB-Stick in den Plattenspieler und den rullino - den Film für Analogkameras - in die Digitalkamera einzulegen versuchen, was dann in einem futuristischen Aufruf mündet: „È finita l'Italia del rullino!“‘

(8) [...] nel 2014 aggrapparsi a una norma del 1970 [...] è come pensare di prendere un i-Phone e di dire 'Dove lo metto il gettone del telefono?' E come pensare che prendere un giradischi e metterci la chiavetta usb pensare, pensare oggi di utilizzare l'articolo 18 come la nostra battaglia e prendere una macchina fotografica digitale e cercare di inserirci un rullino. È finita l'Italia del rullino! Rivendico per la sinistra l'Italia del digitale, l'Italia del domani!

(Leopolda-Rede, 2014)

Wie Bsp. 8 zeigt, durchzieht den Reformdiskurs eine Variante des Zeitwandel-Topos, der im bereits erwähnten Joint paper von Blair und Schröder und den anderen reformbezogenen Reden und Interviews um die Jahrtausendwende auftritt: „Having the same job for life is a thing of the past.“ - „Ein einziger Arbeitsplatz fürs ganze Leben ist Vergangenheit.“ In die politische Argumentation um den Jobs Act wird der Zeitwandel-Topos ebenfalls durch verschiedene Persuasionsstrategien eingebracht (Bsp. 9), die ihn als allseits bekannte und

\footnotetext{
${ }^{16}$ Es handelt sich um Artikel 18 („,Reintegrazione nel posto di lavoro”) des Arbeitnehmerstatuts von 1970
} (Statuto dei lavoratori. Legge 20.05.1970, $\mathrm{n}^{\circ} 300$ ). 
unerschütterliche Tatsache darstellen (z. B. Präsuppositionen, Geminatio, Anadiplose). Dadurch entsteht im beobachteten Fall der doppelte Widerspruch, dass in einem Land, in dem zum Zeitpunkt der zitierten Reden nach offiziellen Statistiken noch über $50 \%$ der Berufstätigen eine Festanstellung hatten, ${ }^{17}$ der Reformdiskurs sich auf das Postulat „Non c'è più il posto fisso"stützt - um ebendiesen abzuschaffen.

(9) Non c'è più il posto fisso, non perché l'abbiamo scelto noi. Non c'è più posto fisso perché è cambiato il mondo [...] è cambiato il pit stop, è cambiato in un arco di tempo strettissimo tutto ciò che ci circonda sul mondo del lavoro.

(Leopolda-Rede, 2014)

$\mathrm{Zu}$ den Persuasionsmitteln mit reformlegitimierendem Potenzial gehören außerdem noch eine Reihe von prädizierenden Syntagmen und Komposita (cf. Busse 2009) wie beschäftigungspolitischer Stillstand, Beschäftigungsstillstand, soziale Hängematte, die erwähnten, auch auf höchster politischer Ebene reproduzierten Bildungen wie Melkkuh Sozialstaat sowie die Metaphern, die auf dem Organismustopos beruhen (z. B. Deutschland vor der Agenda 2010 als der kranke Mann Europas).

\subsection{Der Unternehmensdiskurs}

Der unternehmerische Flexibilisierungsdiskurs manifestiert sich in verschiedenen schriftlichen und mündlichen Textsorten, von Geschäfts- und Nachhaltigkeitsberichten über Pressemitteilungen bis hin $\mathrm{zu}$ Ratgebern für Personalmanager und Bewerber, Stellenangeboten und Werbeanzeigen. So kreisen die Wunschattribute der potenziellen Stellenbewerber um das eingangs skizzierte Idealbild des flexiblen, vielseitigen, lernfähigen, motivierten und möglichst rund um die Uhr einsetzbaren Mitarbeiters (cf. die Stellenanzeigen in Bsp. 10-12).

(10) Cerchiamo persone dinamiche, flessibili e pro-attive, fortemente motivate e focalizzate al raggiungimento degli obiettivi, personali e di gruppo. ${ }^{18}$

(11) Sie verfügen über eine schnelle Auffassungsgabe und lieben die Herausforderung flexibel und vielseitig tätig zu sein?

(12) Nous cherchons des coursiers en vélo flexibles, motivés et souriants! Nous livrons dans Paris de $9 \mathrm{~h}$ à minuit, $7 / 7 \mathrm{j}$.

Gleichzeitig werden die Anstrengungen und Ungewissheiten der Arbeitssuche in Bewerbungsratgebern durch den Hinweis auf unbegrenzte berufliche Aussichten abgefedert (Bsp. 13). Berufe mit niedrigem sozialen Prestige werden in Stellenanzeigen sowie anderen Textsorten aufgewertet: etwa durch positiv oder neutral konnotierte (dt. Raumpflegepersonal) und bedeutungsextensive Lexeme (it. agente, operatore, assistente, tecnico, fr. agent, opérateur usw.) oder durch Anglizismen (dt. Facility Manager). Die daraus resultierende

\footnotetext{
${ }^{17}$ Laut dem Bericht des Statistikamtes ISTAT Il mercato del lavoro negli anni della crisi. Dinamiche e divari (2014) beträgt der Anteil der Dipendenti a tempo pieno (Subkategorie der Kategorie Standard, die tempo pieno e indeterminato beinhaltet) 58,1\% für Männer und 48,1\% für Frauen (S. 89, Tavola 3.3; www.istat.it/it/files/2014/05/cap3.pdf) [22.06.2019].

18 Quellen: (10): http://offerte-di-lavoro.monster.it; (11): www.roberthalf.de/job/essen/sachbearbeiterkreditorenbuchhaltung-wm/149386-DEde; (12): www.cornerjob.com/fr/i/nous-cherchons-des-coursiers-veloflexibles-motives-souriants-nous-livrons-dans-paris-G4UZXfjJPa [05.07.2017].
} 
Bedeutungsvagheit lässt sich zwar im Sinne des auch sonst tendenziell positiv konnotierten und ,politisch korrekten“ unternehmerischen Wording interpretieren; wichtiger in Bezug auf die hier untersuchten Trends ist jedoch, dass sie implizit auch die Perspektive eines Aufstiegs zulässt, die bei näher spezifizierten Bezeichnungen nicht gegeben ist. $\mathrm{Zu}$ erwähnen ist in diesem knappen Überblick noch der Begriff self-management (dt. auch Selbstmanagement, fr. autogestion, automanagement), der mit steigender Frequenz in Ratgebern und Bildungsangeboten verschiedener Institutionen auftritt (Bsp. 14) und den erwähnten Imperativ der permanenten Selbstoptimierung widerspiegelt.

(13) Il mercato del lavoro è oggi sempre più dinamico e richiede ai lavoratori flessibilità e mobilità. Se da un lato questo presuppone un grande impegno e una maggiore iniziativa personale, offre comunque, dall'altro lato, prospettive più ampie. ${ }^{19}$

(14) Die Seminare Existenzgründung für Übersetzer und Dolmetscher und Zeit- und Selbstmanagement können zu einem Vorzugspreis auch gemeinsam gebucht werden.

Mit Blick auf den Stellenabbau als eines der tragenden Elemente einer flexibilisierenden Personalpolitik können exemplarisch mehrere einzelsprachenübergreifende Tendenzen erwähnt werden: lexikalische und syntaktische Euphemisierungsstrategien, Toposeinsatz, Diskurshybridisierung. Die Euphemisierung wird dabei als Oberbegriff für eine Reihe von lexikalisch-semantischen und syntaktischen Mitteln und Strategien benutzt, für die im gegebenen pragmatisch-kommunikativen Rahmen eine verhüllende, verschleiernde, abschwächende, beschönigende, Bezeichnungsnot lindernde oder Elemente des jeweils aktivierten Sinnhorizonts ausblendende (Wengeler 1992: 65) Funktion angenommen werden kann (Rocco 2015a). Dazu gehört mit Blick auf den Unternehmensdiskurs z. B. der bedeutungsgeneralisierende bzw. abstrahierende Gebrauch lexikalischer Mittel: So begegnet man in Unternehmensberichten sowie Pressemitteilungen Ausdrücken wie restructuring, Restrukturierung, Umstrukturierung, ristrutturazione, Personalanpassung (mit teilweise variierenden Gebrauchskonnotationen), die im gegebenen Kontext euphemistisch für den Stellenabbau eingesetzt werden können, andererseits aber nicht unbedingt eine (z. T. im Zusammenhang mit den Euphemismen postulierte) „verschleiernde” Wirkung ${ }^{20}$ haben müssen. Die potenzielle Vielschichtigkeit der Illokutionen und Perlokutionen lässt sich am Syntagma Verlagerung der Arbeitsplätze, eng. relocation of jobs veranschaulichen. Der Bestandteil verlagern bzw. relocate blendet einzelne Aspekte des aktivierten Sinnhorizonts aus, insofern aus der Perspektive des Unternehmens eine Beschränkung auf die Tatsache möglich ist, dass sich die produzierende Instanz nicht mehr am Ort A, sondern am Ort B befindet, also in diesem Sinne von A nach B verlagert wurde. Aus der Beschäftigtenperspektive steht hingegen der Umstand im Vordergrund, dass die Stelle am Ort A abgeschafft und am Ort B neu geschaffen - und möglicherweise anderweitig besetzt wurde, was konkret für die am Ort A beschäftigten Individuen, die nicht an den Ort B wechseln können, nicht Verlagerung, sondern Entlassung bedeutet. In dieser Hinsicht könnte

\footnotetext{
${ }^{19}$ Quellen: (13): Ufficio Regionale di Collocamento: "Come presentare und buona candidatura", www.eures.ch/it/serveattachment/1c682e400bdb11e3b4e561c36ef204670467/seco_broschuere_i_low.pdf; (14): http://seminare.bdue.de/2768 [05.07.2017].

${ }^{20}$ Die Opposition verschleiernd-verhüllend findet man in verschiedenen Varianten bei Bohlen (1994: 33, 169) Luchtenberg (1985: 24), Rada (2001: 64f.) und Zöllner (1997: 159). Zur Schwierigkeit der Abgrenzung zwischen verschleiern, verhüllen und anderen möglichen Funktionen cf. Rocco (2017).
} 
für bestimmte Textsorten und Situationen eine ursprünglich euphemistische Intention von verlagern angenommen werden; für eine Reihe anderer möglicher Kontexte ist dagegen nicht von imagestrategischer Euphemisierung, sondern vielmehr von fachinternem oder technischem Gebrauch auszugehen. Der Ausdruck verlagern ist also, illokutiv und perlokutiv, in hoch spezialisierten Kapiteln von Geschäftsberichten anders einzuordnen als etwa in Pressemitteilungen an die Öffentlichkeit. In diesem Sinne ist abschließend zu betonen, dass keine Sprachmittel a priori und ohne Berücksichtigung von Textfunktionen, Akteuren, Mikround Makrokontext und anderen pragmatisch-kommunikativen Aspekten als euphemistisch oder dysphemistisch einstufbar sind.

Die Stellenstreichung wird in den imagerelevanten Teilen von Geschäfts- und Nachhaltigkeitsberichten oft als schmerzhafte, aber notwendige, unvermeidbare oder unvermeidliche Kostensenkungsmaßnahme präsentiert, die so sozialverträglich wie möglich verläuft. ${ }^{21}$ Der rücksichtsvolle Grundton wird also mit dem Argument der alternativlosen bzw. überlebensnotwendigen Einsparung kombiniert:

(14) Im Rahmen der fusionsbedingten Anpassungen der Prozesse und des Abbaus von Doppelarbeit musste darüber hinaus auch ein Personalabbau vorgenommen werden, der jedoch möglichst sozialverträglich gestaltet wurde.

(Geschäftsbericht DZ 2004: 11)

(15) Bei allen Maßnahmen werden die Belange und Bedürfnisse der Mitarbeiter weitest möglich berücksichtigt. Unvermeidbarer Personalabbau erfolgt zügig und sozialvertraglich.

(Geschäftsbericht BLB 2008: 27)

(16) (...) für den notwendigen Abbau von Sach- und Personalkosten, bei dem wir im Rahmen unserer Planungen liegen. So haben wir seit Beginn der Restrukturierung (...) bereits Austritte von rund 2000 Vollzeitstellen vereinbart.

(Geschäftsbericht LBBW 2011:3)

Bezeichnend ist, dass der Topos „geringere Kosten = höhere Effizienz“ normalerweise Stellenabbau-Argumentationen wie „Der Personalabbau lässt die Personalkosten sinken und ist deshalb effizient" untermauert, derselbe Topos allerdings auch bei Gegenargumentationen herangezogen werden könnte, etwa: „Personalabbau verursacht (zumindest kurzfristig) hohe Kosten (durch Abfindungen etc.) und ist deshalb nicht effizient“" (Hossfeld 2011: 79).

Aus syntaktischer Sicht sind in diesem Zusammenhang Verfahren der Detransitivierung und Deagentivierung von Interesse, die in den imagerelevanten unternehmerischen Texten und Subtexten beobachtet werden können. So tritt das Unternehmen tendenziell als das Agens auf, als Träger strategisch wichtiger, gewinnbringender Handlungen (wir fördern Nachwuchskräfte, wir gewinnen Talente, wir bieten Karrierechancen, wir unterstützen Mitarbeiter in schwierigen Zeiten; cf. Rocco/Canavese 2016), während dem kollektiven Akteur - den Mitarbeitern - i. d. R. die Rolle des Benefiziärs der Unternehmensangebote oder Adressaten der Dankesäußerungen zufällt. In den Stellenabbau-Passagen tritt hingegen das Unternehmen als Agens zurück, bei gleichzeitig steigender Häufigkeit intransitiver und reziproker Verben und deverbaler Ableitungen bzw. von Konstruktionen mit anderen Handlungsträgern: So kommt es zu Austritten (Bsp. 16) und Trennungen (Bsp. 17), in anderen

${ }^{21}$ Für weitere Beispiele cf. Rocco/Canavese (2016: 143f.). 
Texten zu wegfallenden Stellen und austretenden Mitarbeitern. Die Vielfalt der potenziellen Bedeutungsnuancen des Begriffs Trennung, der in personalbezogenen Texten verschiedener Sprachen auftritt (separation, séparation, separazione) kann in Bsp. 17 genauer betrachtet werden: Bei den ersten beiden Vorkommen von Trennung beinhaltet das zugrunde liegende Verb tatsächlich noch das Merkmal der Reziprozität, während beim letzten Vorkommen (Konditionen für eine einvernehmliche Trennung) die Wiederholung und das Adjektiv einvernehmlich euphemistisch eine Reziprozität suggerieren, die mit Blick auf die ungleichen Verhandlungspositionen und auf den Inhalt des letzten Satzes nicht unbedingt gegeben ist.

(17) Für Mitarbeiter, die von sich aus ernsthaftes Interesse an einer einvernehmlichen Trennung haben, wird eine Info-Stelle eingerichtet, die unverbindlich über individuelle Abfindungspakete informiert. Soweit auf dieser Basis eine Trennung in Frage kommt, wird den Mitarbeitern ein konkretes Trennungsangebot unterbreitet.

Diejenigen Mitarbeiter, die seitens der Bank für einen Abbau vorgesehen sind, werden unmittelbar angesprochen und die Konditionen für eine einvernehmliche Trennung diskutiert. Als Ultima Ratio können betriebsbedingte Kündigungen zur Erreichung der erforderlichen Abbauziele nicht ausgeschlossen werden.

(Geschäftsbericht BLB 2008, S. 27)

Besonders häufig ist von Trennung und Trennungsmanagement ${ }^{22}$ in der blühenden Ratgeberliteratur für Personalmanager die Rede. Hier erweist sich Trennung als äußerst produktiv - so ergab die exemplarische Auswertung des Inhaltsverzeichnisses eines Personalratgebers mit dem Titel „Trennungskultur und Mitarbeiterbindung“ (Andrzejewski/Refisch 2015) eine Reihe von Bildungen, die hier zur besseren Anschaulichkeit im Rahmen der jeweiligen Abschnittsüberschriften zitiert werden: „2.1 Die Not der Vorgesetzten im Trennungsgespräch“, ,5.1.4 Trennungs-Kultur als Kostenbremse“, „5.2.2 Inhalte des Trennungspakets“, „6.3 Qualifizierung für Trennungen international“, „7 Zukunft der Trennungs-Kultur“, „9 Organisation von Trennungsprojekten", „13.2.2 Trennungsbotschaft aussprechen“. Die folgenden Beispiele entstammen hingegen der „Sieben Gedanken zum Trennungsmanagement" betitelten Zusammenfassung des Interviews mit einer Unternehmensberaterin, die als Kündigungs-Coach bzw. Trennungscoach „Unternehmen rund um die faire und wirtschaftliche Trennung von Mitarbeitern“ berät: „Erfolgreiche Trennungsprozesse haben ein $\mathrm{A}$ und ein $\mathrm{Z}$ [...] Besser als Aktionismus ist ein gut durchdachter Trennungsprozess. [...] Aber bleiben Sie konsequent bei Ihrer Trennungsentscheidung.“ (Walker 2014).

Zum Abschluss sei noch auf die Tendenz zur Diskurshybridisierung im Sinne der Verschränkung eines utilitaristisch-gewinnorientierten, mit Controlling-Elementen durchsetzten Personaldiskurses mit humanistisch und postmaterialistisch geprägten Werten wie Toleranz, Chancengleichheit, Freiheit, Respekt und Lebensqualität hingewiesen. Das sich darin reflektierende Prinzip der Ökonomisierung der Ethik bzw. „Ethisierung” der Ökonomie (Schwartz 2007) lässt sich an Leitsätzen wie „Gender Diversity lohnt sich“ (Bayerische

\footnotetext{
22 Trennungsmanagement entspricht dem englischen separation management. Im Französischen ist in vergleichbaren Textsorten der Begriff gestion de séparation belegt; im Italienischen ist er aktuell am schwächsten vertreten, was angesichts der Dynamik der Entwicklungen und der Bedeutung englischsprachiger Vorbilder (cf. auch Diversity Management) eher als vorübergehender Stand einzuschätzen ist.
} 
Landesbank, Geschäftsbericht 2012) veranschaulichen, sowie am folgenden Beispiel aus dem Geschäftsbericht der Deutschen Bank, die sich schon vergleichsweise früh mit dem DiversityKonzept auseinandersetzte, das inzwischen in großunternehmerischen Geschäfts- und Nachhaltigkeitsberichten mit zunehmender Häufigkeit auftritt. ${ }^{23}$

(18) Vielfalt zu fördern ist Bestandteil unserer Unternehmensidentität. Ihr liegt ein weit gefasstes Verständnis von Diversity zu Grunde. Unsere Denkweise ist geprägt von gegenseitiger Achtung, Offenheit und der Selbstverpflichtung zu ständiger beruflicher und persönlicher Weiterentwicklung. Wir haben diese Grundhaltung in einem für uns weltweit gültigen Verhaltenskodex festgeschrieben, dessen Beachtung wir von jeder Mitarbeiterin und jedem Mitarbeiter erwarten. Wir wollen ein offenes Umfeld bieten, das Kulturkonflikte vermeidet und in dem die Mitarbeiter nach ihren Leistungen gefördert werden.

(Geschäftsbericht DB 2002, S. 50)

\section{$3 \quad$ Schlussbetrachtung}

Im vorgegebenen Rahmen konnten einige persuasive Strategien des Flexibilisierungsdiskurses nur kurz und ohne Anspruch auf Vollständigkeit umrissen werden. Es lassen sich trotzdem Tendenzen erkennen, deren vertiefende und systematische Untersuchung auch für andere Bereiche des eingangs skizzierten wirtschaftspolitischen Paradigmenwechsels von Interesse sein könnten (z. B. epilinguistischer und akademischer bzw. wissensbezogener Diskurs). So lässt das in den flexibilisierungsbezogenen Argumentationen oft betonte Allgemeininteresse, das, wie gezeigt wurde, sowohl in politischen als auch in unternehmerischen Texten thematisiert wird, hegemoniale Diskursformationen annehmen. Diese zeichnen sich dadurch aus, dass in ihnen glaubwürdig das Gemeinwohl oder ein korrespondierendes Konzept mit dem Anspruch, das Allgemeine zu vertreten, im Zentrum steht (Nonhoff 2014: 193), ein leeres Signifikant im Sinne von Laclau (2002: 65-78). In diesem Sinne wäre aus diskurs- und persuasionsanalytischer Sicht die Frage zu vertiefen, ob und inwiefern „Flexibilität“ und „Mobilität“ (neben „Diversity“, „Nachhaltigkeit“ usw.) als leere Signifikanten fungieren, als diskursmarkierende und überparteilich konsensfähige Hochwertbegriffe (Burkhardt 1998: 103), über die partikulare Ziele als universelle vermittelt werden. Ein methodisches Desiderat bei der Erforschung dieser oder anderer relevanter Fragen wäre eine systematische, auf repräsentativen Korpora beruhende Herangehensweise, die neben in der Diskurs- und Persuasionsforschung bereits viel beachteten Textsorten wie Pressetexten, Forumsbeiträgen und Politikerreden auch andere, stark unterrepräsentierte Textsorten einbezieht und eine sprachübergreifende, nach Möglichkeit diskursvergleichende Perspektive einnimmt.

\section{Literaturverzeichnis}

Accornero, Aris (2001): „Dal fordismo al post-fordismo: il lavoro e i lavori“. Rassegna Sindacale 1. www.ossimoro.it/lavoro1.html [23.06.2019].

Angermuller, Johannes et al. (eds.) (2014): Diskursforschung: Ein interdisziplinäres Handbuch, Bd. 2, Cambridge etc.: Cambridge University Press.

Angermuller, Johannes/Maeße, Jens (2014): „Der Hochschulreformdiskurs. Thema, Gegenstand, Korpus“. In: Angermuller, Johannes et al. (eds.): Diskursforschung. Ein

\footnotetext{
${ }^{23}$ Ausführlicher dazu Rocco/Canavese (2016).
} 
interdisziplinäres Handbuch. Band 2. Methoden und Praxis der Diskursanalyse. Perspektiven auf Hochschulreformdiskurse. Bielefeld, transcript: 23-36.

Bartoszewicz, Iwona (2008): Formen der Persuasion im deutsch-polnischen politischen Dialog: Untersuchungen zu politischen Reden zwischen 1989 und 1995. Wroclaw: Acta Universitatis Wratislaviensis.

Bascetta, Marco et al. (2015): Economia politica della promessa. Roma: Manifestolibri.

Blommaert, Jan (2005): Discourse. Cambridge: Cambridge University Press.

Bohlen, Aandreas (1994): Die sanfte Offensive. Untersuchungen zur Verwendung politischer Euphemismen in britischen und amerikanischen Printmedien bei der Berichterstattung über den Golfkrieg im Spannungsfeld zwischen Verwendung und Mißbrauch der Sprache. New York etc.: Lang.

Bosančić, Saša (2014): Arbeiter ohne Eigenschaften. Über die Subjektivierungsweisen angelernter Arbeiter. Wiesbaden: VS

Bourdieu, Pierre (1984): Questions de sociologie. Paris: Les Editions de Minuit.

Brand, Karl-Werner (2017): „Naturschutz - Umweltschutz - Nachhaltige Entwicklung: Transformation gesellschaftlicher Regulationsmodelle und sozial-ökologischer Regime“" In: Brand, Karl-Werner (ed.) Die sozial-ökologische Transformation der Welt. Ein Handbuch. Frankfurt a. M./New York, Campus: 79-116.

Bröckling, Urlich (2007): Das unternehmerische Selbst: Soziologie einer Subjektivierungsform. Frankfurt a. M.: Suhrkamp.

Brygo, Julien/Cyran, Olivier (2016): „Direction des Ressources Heureuses“. Le Monde diplomatique, octobre 2016, 28.

Burkhardt, Armin (1998): „Deutsche Sprachgeschichte und politische Geschichte“. In: Besch, Werner et al. (eds.): Sprachgeschichte. Ein Handbuch zur Geschichte der deutschen Sprache und ihrer Erforschung. Bd. 1. Vollst. neu bearb. u. erw. Aufl. Berlin/New York, de Gruyter: 98-122.

Busse, Dietrich/Teubert, Wolfgang (1994) „Ist Diskurs ein sprachwissenschaftliches Objekt? Zur Methodenfrage der Historischen Semantik“. In: Teubert Wolfgang/Busse Dietrich (eds.): Begriffsgeschichte und Diskursgeschichte. Methodenfragen und Forschungsergebnisse der historischen Semantik. Opladen, Westdeutscher Verlag: 10-28.

Busse, Dietrich (2009): „Prädikation durch Wortbildung. Zum Zusammenhang von Wortgrammatik und Satzsemantik“". In: Liebert, Wolf-Andreas/Schwinn, Horst (eds.): Mit Bezug auf Sprache. Festschrift für Rainer Wimmer. Tübingen, Narr: 485-505.

Butterwegge, Christoph/Hickel, Rudolf/Ptak, Ralf (1998): Sozialstaat und neoliberale Hegemonie. Berlin: Elefantenpress.

Candeias, Mario (2004): Neoliberalismus, Hochtechnologie, Hegemonie, Argument. Hamburg: Argument.

Chassé, Daniel S. (2016): „Gab es in der Schweiz eine neoliberale Wende? Ein Kommentar.“ In: Criblez, Lucien/Rothen, Christina/Ruoss, Thomas (eds.): Staatlichkeit in der Schweiz. Regieren und verwalten vor der neoliberalen Wende. Zürich, Chronos Verlag: 369-384.

Chomsky, Noam (2003): Profit over People - Neoliberalismus und globale Weltordnung. Hamburg: Europa-Verlag. 
Dörre, Klaus et al. (2008): „Eigensinnige 'Kunden'. Der Einfluss strenger Zumutbarkeit auf die Erwerbsorientierung Arbeitsloser und prekär Beschäftigter". SFB 580 Mitteilungen 26: $10-36$.

Dörre, Klaus et al. (2013): Bewährungsproben für die Unterschicht? Soziale Folgen aktivierender Arbeitsmarktpolitik. Frankfurt/New York: Campus.

Drommler, Michael/Kuck, Kristin (2013): „Krise aus Metaphern - Krise in Metaphern. Metaphorische Konstruktionen von Krisenkonzepten am Beispiel der Debatten zur 'Agenda 2010' und zur 'Finanzkrise 2008/09““. In: Wengeler, Martin/Ziem, Alexander (eds.): Sprachliche Konstruktionen von Krisen. Interdisziplinäre Perspektiven auf ein fortwährend aktuelles Phänomen. Bremen, Hempgen: 209-239.

Elster, Frank (2007): Der Arbeitskraftunternehmer und seine Bildung. Zur (berufs-) pädagogischen Sicht auf die Paradoxien subjektivierter Arbeit. Bielefeld: transcript.

Fairclough, Norman (2011): „Globaler Kapitalismus und kritisches Diskursbewußtsein“. In: Reiner Keller et al. (eds.): Handbuch Sozialwissenschaftiche Diskursanalyse. Opladen, Leske + Budrich: 335-351.

Flores, Nelson (2013): „The unexamined relationship between neoliberalism and plurilingualism: A cautionary tale“. TESOL Quarterly 47(3): 500-520.

Fumagalli, Andrea (2016): „'Jobs Act', le grand bluff de Matteo Renzi.“ Le Monde diplomatique, juillet 2016: 8-9. www.monde-diplomatique.fr/2016/07/FUMAGALLI/55955. [05.07.2017].

Glauser, Laura (2016): Das Projekt des unternehmerischen Selbst. Eine Feldforschung in der Coachingzone. Bielefeld: transcript.

Hegelich, Simon/Knollmann, David/Kuhlmann, Johanna (2011): Agenda 2010: Strategien Entscheidungen - Konsequenzen. Wiesbaden: VS Verlag.

Heller, Monica (2010): „The commodification of language“. The Annual Review of Anthropology 39: 101-114.

Hermanns, Fritz (1989): „Deontische Tautologien. Ein linguistischer Beitrag zur Interpretation des Godesberger Programms der SPD“. In: Klein, Josef (ed.): Politische Semantik. Opladen, Westdeutscher Verlag: 69-149.

Holmes, Brian (2002): „The Flexible Personality. For a New Cultural Critique“. In: transversal- eipcp multilingual webjournal. http://eipcp.net/transversal/1106/holmes/en/base_edit. [22.06.2019].

Hossfeld, Heiko (2011): Sprachliche Legitimation von Personalabbaumaßnahmen. Eine Argumentations- und Metaphernanalyse der Massenkommiunikation zweier Großbanken. München/ Mering: Hampp. (= Schriftenreihe empirische Personal- und Organisationsforschung 50).

Joseph, Jonathan (2013): „Resilience as embedded neoliberalism: a governmentality approach“. Resilience 1/1: 38-52.

Klein, Josef (2007): „Linguistische Hermeneutik politischer Rede. Eine Modellanalyse am Beispiel von Kanzler Schroders Verkundigung der Agenda 2010“. In: Hermanns, Fritz/Holly, Werner (eds.): Linguistische Hermeneutik und Praxis des Verstehens und Interpretierens. Tübingen, Niemeyer: 201-238. 
Kubota, Ryuko (2015): „Inequalities of Englishes, English Speakers, and Languages: A Critical Perspective on Pluralist Approaches to English“. In: Tupas, Ruanni: Unequal Englishes: The Politics of Englishes Today. London, Palgrave Macmillan: 21-41.

Kuhlen, Rainer (2002): „Napsterisierung und Venterisierung Bausteine zu einer politischen Ökonomie des Wissens“. PROKLA - Zeitschrift für kritische Sozialwissenschaft 126/2002, $57-88$.

Laclau, Ernesto (2002): „Was haben leere Signifikanten mit Politik zu tun?“. In: Laclau, Ernesto (ed.) Emanzipation und Differenz. Wien, Turia + Kant: 65-78.

Lausberg, Heinrich (1973): Handbuch der literarischen Rhetorik. München: Hueber.

Luchtenberg, Sigrid (1985): Euphemismen im heutigen Deutsch. Mit einem Exkurs zu Deutsch als Fremdsprache. Frankfurt a. M. etc.: Lang.

Lüdi, Georges (2007): „Sprachverhalten, Sprachpolitik, Diskurs über Sprache: Staatlichkeit in Europa zwischen dem einsprachigen Nationalstaat und dem mehrsprachigen Vielvölkerstaat"،. In: Nekula, Marek/Fleischmann, Ingrid/Greule, Albrecht (eds.): Franz Kafka im sprachnationalen Kontext seiner Zeit: Sprache und nationale Identität in öffentlichen Institutionen der böhmischen Länder. Köln etc., Böhlau: 13-30.

Marchart, Oliver (2013): Die Prekarisierungsgesellschaft: Prekäre Proteste. Politik und Ökonomie im Zeichen der Prekarisierung. Bielefeld: transcript.

Mattoni, Alice (2016): Media Practices and Protest Politics: How Precarious Workers Mobilise. London/New York: Routledge.

Mortara Garavelli, Bice (2008): Manuale di retorica. Milano: Bompiani.

Mulholland, Joan (1994): Handbook of Persuasive Tactics: A practical language guide. London: Routledge.

Nonhoff, Martin/Herschinger, Eva (2014): „Einleitung: Methoden und Analysepraxis. Ein kaleidoskopartiger Blick auf Hochschulreformdiskurse“. In: Angermuller, Johannes et al. (eds.): Angermuller, Johannes et al. (eds.): Diskursforschung. Ein interdisziplinäres Handbuch. Band 2. Methoden und Praxis der Diskursanalyse. Perspektiven auf Hochschulreformdiskurse. Bielefeld, transcript: 15-22.

Nollert, Michael/Pelizzari, Alessandro (2008) „Flexibilisierung des Arbeitsmarktes als Chance oder Risiko? Atypisch Beschäftigte in der Schweiz“. In: Szydlik, Marc (ed.): Flexibilisierung: Folgen für Arbeit und Familie. Wiesbaden, Verlag für Sozialwissenschaften: 130-148.

Nullmeier, Frank (2004): „Vermarktlichung des Sozialstaats“. WSI Mitteilungen 9/2004: 495-500.

Oschmiansky, Frank (2010): Aktivierender Staat und aktivierende Arbeitsmarktpolitik. www.bpb.de [05.07.2017].

Piller, Ingrid/Cho, Jinhyun. (2013): „Neoliberalism as language policy“. Language in Society 42(1), 23-44.

Polanyi, Karl (1978): The Great Transformation. Frankfurt a. M.: Suhrkamp.

Pongratz, Hans J./Voß, Günter G. (2003): From employee to 'entreployee': Towards a 'selfentrepreneurial' work force? In: Concepts and Transformation 8/3: 239-254.

Promberger, Markus (2012): Topographie der Leiharbeit: Flexibilität und Prekarität einer atypischen Beschäftigungsform. Berlin: Sigma. 
Rada, Roberta (2001): Tabus und Euphemismen in der deutschen Gegenwartssprache; mit besonderer Berücksichtigung der Eigenschaften von Euphemismen. Budapest: Akad. Kiadó.

Rocco, Goranka (2015a): „Euphemismen und Dysphemismen im Flexibilisierungsdiskurs. Auf dem Weg zu einem mehrperspektivischen Untersuchungsdesign". Lingue e Linguaggi 13: $257-275$.

Rocco, Goranka (2015b): „Politische Inszenierung der Flexibilisierung aus diskurslinguistischer Sicht". In: Hennemann, Anja/Schlaak, Claudia (eds.): Politische und mediale Diskurse. Fallstudien aus der Romania. Berlin, Fran \& Timme: 83-111.

Rocco, Goranka/Canavese, Paolo (2016): „Versprachlichung unternehmerischer Beschäftigungspolitik in Geschäftsberichten". In: Reinmuth, Marcus et al. (eds.): Unternehmenskommunikation für Banken und Versicherer: Krisen bewältigen, Vertrauen schaffen. Stuttgart, Schäffer-Poeschel: 127-148.

Rocco, Goranka (2017): „Politische Korrektheit und sprachliche Höflichkeit als komplexitätsreduzierende Rituale der Wirtschaftskommunikation". In: Ehrhardt, Claus/Neuland, Eva (eds.): Sprachliche Höflichkeit. Historische, aktuelle und künftige Perspektiven. Tübingen, Narr: 385-399.

Rocco, Goranka/Schafroth, Elmar (2019): „Diskurse im interlingualen Vergleich: Forschungsperspektiven und methodische Herausforderungen". In: Rocco, Goranka/Schafroth, Elmar (eds.): Vergleichende Diskurslinguistik: Methoden und Forschungspraxis. Frankfurt a. M. etc.: Lang.

Schröter, Melani (2004): „Ruck-Rede oder Rucklein-Lyrik. Wie 'rhetorisch' war die 'Ruckund Reformrede' Schroders vom 14. Marz 2003?" Sprachreport 2/2004: 20-27.

Schwartz, Thomas (2007): „Ökonomisierung der Ethik oder »Ethisierung« der Ökonomie? Überlegungen zur wissenschaftstheoretischen Relevanz von CSR-Konzepten“. In: Gramlich, Dieter/Träger, Manfred (eds.): Herausforderungen einer zukunftsorientierten Unternehmenspolitik: Ökonomie, Umwelt, Technik und Gesellschaft als Determinanten. Wiesbaden, DUV: 91-105.

Slaby, Jan (2016): „Kritik der Resilienz“. In: Kurbacher, Frauke A. /Wüschner, Philipp (eds.): Was ist Haltung? Würzburg, Königshausen \& Neumann: 273-298.

Spira, Andreas (1981): „Wert und Unwert der Topik“. In: Breuer, Dieter/Schanze, Helmut (eds.): Topik. Beiträge zur interdisziplinären Diskussion. München, Fink: 42-53.

Stark, Christopher (2014): Neoliberalyse - Über die Ökonomisierung unseres Alltags. Wien: Mandelbaum.

Teubert, Wolfgang (1998a): „Korpus und Neologie“. In: Teubert, Wolfgang (ed.): Neologie und Korpus. Tübingen, Narr: 129-170.

Teubert, Wolfgang (1998b): „Eigentum, Arbeit, Naturrecht. Schlüsselwörter der Soziallehre im Wandel“. In: Kämper Heidrun/Schmidt, Hartmut (eds.): Das 20. Jahrhundert. Sprachgeschichte - Zeitgeschichte. Berlin/New York, de Gruyter: 188-124.

Tickell, Adam/Peck, Jamie A. (1995): „Social regulation after Fordism: regulation theory, neo-liberalism and the global-local nexus". Economy and Society 24/3: 357-386.

Toulmin, Stephen (1996): Der Gebrauch von Argumenten. Weinheim: Beltz Athenäum.

Trube, Achim (2004): „Die neue deutsche Arbeitsmarktpolitik und der Wandel des Sozialstaats". Sozialer Fortschritt 3: 62-82. 
Varoufakis, Yanis/Chomsky, Noam (2016): Full transcript of the Yanis Varoufakis|Noam Chomsky NYPL discussion. yanisvaroufakis.eu/2016/06/28/full-transcript-of-the-yanisvaroufakis-noam-chomsky-nypl-discussion [05.07.2017].

Walker, Dagmar (2014): „Sieben Gedanken zum Trennungsmanagement.“ humanresourcesmanager.de/news/sieben-gedanken-zum-trennungsmanagement.html [21.06.2019].

Wengeler, Martin (1992): Die Sprache der Aufrüstung. Zur Geschichte der Rüstungsdiskussionen nach 1945. Opladen: Westdeutscher Verlag.

Wengeler, Martin (1997): „Vom Nutzen der Argumentationsanalyse für eine linguistische Diskursgeschichte. Konzept eines Forschungsvorhabens“. Sprache und Literatur in Wissenschaft und Unterricht 80: 96-109.

Wengeler, Martin (2013): „Von ist ein Begriffsbastard' bis 'Steuern runter macht Deutschland munter'. Zur Geschichte von Wirtschaftsdiskursen im 20. Jahrhundert". Sprachwissenschaft 38: 71-99.

Zöllner, Nicole (1997): Der Euphemismus im alltäglichen und politischen Sprachgebrauch des Englischen. Frankfurt a. M. etc.: Lang.

\section{Quellen der Beispiele aus den analysierten Texten}

Andrzejewski, Laurenz/Refisch, Hermann (2015): Trennungskultur und Mitarbeiterbindung. Neuwied: Luchterhand.

Blair, Tony/Schröder, Gerhard (1999): Europe: The Third Way - Die Neue Mitte. London: Labour Party.

Bundesministerium für Wirtschaft und Arbeit (2005): Vorrang für die Anständigen - Gegen Missbrauch, ,Abzocke“ und Selbstbedienung im Sozialstaat. Ein Report vom Arbeitsmarkt im Sommer 2005. https://harald-thome.de/fa/harald-thome/files/Gesetzestexte\%20SGB\% 20II\%20+\%20VO/Gesetzestexte\%20SGB $\% 20 X I I \% 20+\% 20 \mathrm{VO} /$ Seminare/Clement/Sozial missbrauch_Bericht_BMWA.pdf[06.08.2019].

Geschäftsbericht der genossenschaftlichen Bank DZ Bank AG für 2004 (DZ 2004). pioneerprvess.ga/content/dzbank_de/de/home/unser_profil/investorrelations/berichte/Archi v.DownloadLink.download.html?download=9VJrEyDqQfoEnfnmYpc8QFy9k6dQjkeFjP1 4JAiFXLWA194dPXkZOqtTzc6YH_xR8O_i1JzwI2HrHcnysipr_nj9VghNF6FXHRR_vZ PTDbsQf-KRwlSrTg [05.07.2017].

Geschäftsbericht der Bayerischen Landesbank für 2008 (BLB 2008). gb08.bayernlb.de/cgibin/show.ssp?companyName=bayernlb\&report_id=gb2008\&language $=$ German\&fallback $=1$ [06.08.2019].

Geschäftsbericht der Landesbank Baden-Württemberg (LBBW 2011). lbbw.de/public/geschaeftsbericht/lbbw_geschaeftsbericht_2011_7xc8gtyj3_m.pdf [06.08.2019].

Harvey, Armin (2012): Hartz IV: Arm am Beutel, krank am Herzen schleppt ich meine langen Tage. Münster: Principal. 